\title{
A case of hypoharyngeal lipoma with extrapharyngeal extension
}

\author{
Ognjen Cukic ${ }^{1 *}$, Milan Jovanovic ${ }^{2}$
}

\begin{abstract}
Hypopharyngeal lipoma is a rare entity and is usually asymptomatic until it reaches a large size. The lipoma described in this case was unusual because of its deep extension from the hypopharynx to the visceral space of the neck. On endoscopic and MRI examination the tumour appeared as an encapsulated, well-defined lesion covered by yellowish intact mucosa. The lipoma was completely removed through the transcervical approach. The surgery produced excellent cosmetic results and no functional impairment. The approach used also provided a rapid and uneventful postoperative recovery and optimal locoregional control of the disease during the follow-up period.
\end{abstract}

Keywords: airway obstruction; hypopharynx; laryngoscopy; lipoma.

\section{Introduction}

${ }^{1}$ Clinical Hospital Centre Zemun, Department of Otorhinolaryngology with Maxillofacial Surgery, Zemun, Serbia

${ }^{2}$ University of Belgrade, School of Medicine, Belgrade, Serbia

Financial support: None. Conflicts of interest: No conflicts of interest declared concerning the publication of this article. Submitted: August 30, 2018. Accepted: February 14, 2019.

The study was carried out at the Department of Otorhinolaryngology with Maxillofacial Surgery, Clinical Hospital Centre "Zemun", Vukova 9, 11080, Zemun, Serbia,

Article Notes: This study was not presented at scientific congresses or meetings.

Copyright Cukic et al. This is an Open Access article distributed under the terms of the Creative Commons Attribution License, which permits unrestricted use, distribution, and reproduction in any medium, provided the original work is properly cited.
Lipomas are the most common benign mesenchymal tumours, originating from adipose tissue. They are usually located on the body and extremities, where adipose tissue is abundant. However, lipomas are less frequently encountered in the head and neck region, where they constitute under $1 \%$ of benign neoplastic pathology of the upper aerodigestive tract.' Lipomas of the hypopharynx are extremely uncommon and their clinical appearance is of a solitary, sessile or pedunculated mass projecting in the pharyngeal lumen, rarely extending to the surrounding spaces. Although histologically benign, large lipomas can cause sudden airway blockage, and therefore immediate surgical removal is recommended.

The aim of this study is to describe a case of large lipoma involving the left piriform sinus with an unusual deep extension to the visceral space of the neck.

\section{Case report}

A 66-year-old male with a three-year history of foreign body sensation in his throat was referred to our outpatient clinic. His medical history was unremarkable and he had no breathing or swallowing difficulties. Mirror laryngoscopy and subsequent direct laryngoscopic examination showed a lobulated submucosal bulking originating from the left posterolateral pharyngeal wall, obliterating the left piriform fossa, and extending upwards to the level of the aryepiglottic fold, partially obstructing the view of the laryngeal 
inlet (Figure 1). No externally visible cervical mass was noted. The magnetic resonance imaging (MRI) demonstrated a well-defined, dumbbell-shaped mass extending posterior and lateral from the hypopharynx to the left visceral space (Figure 2). The mass was completely removed in 'en-bloc' fashion using the transcervical approach (Figure 3). The removal of the hypopharyngeal part of the tumour resulted in a small mucosal defect of the posterolateral aspect of hypopharynx, which was then closed with interrupted resorbable sutures. Microscopic examination demonstrated an encapsulated tumour, composed of mature adipocytes with no cellular atypia which confirmed features of lipoma. The excised tumour measured $8 \mathrm{~cm}$ in diameter at its largest point. The wound was closed in layers and the nasogastric feeding tube was introduced and maintained for the next ten days. The recovery was uneventful, and the patient was discharged after successfully starting oral diet. The patient was free of disease during the one-year follow up.

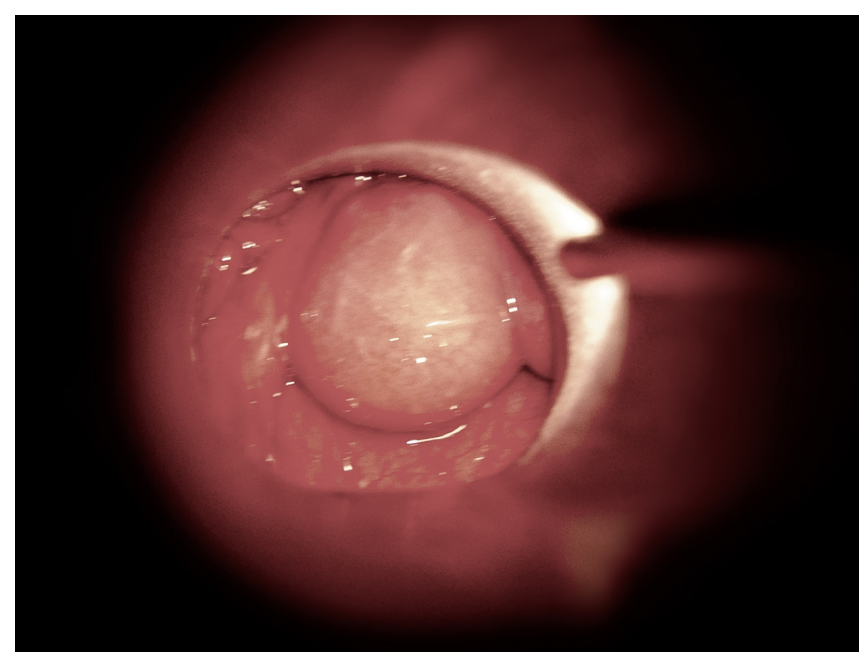

Figure 1. Direct laryngoscopy of the patient with the hypopharyngeal lipoma.

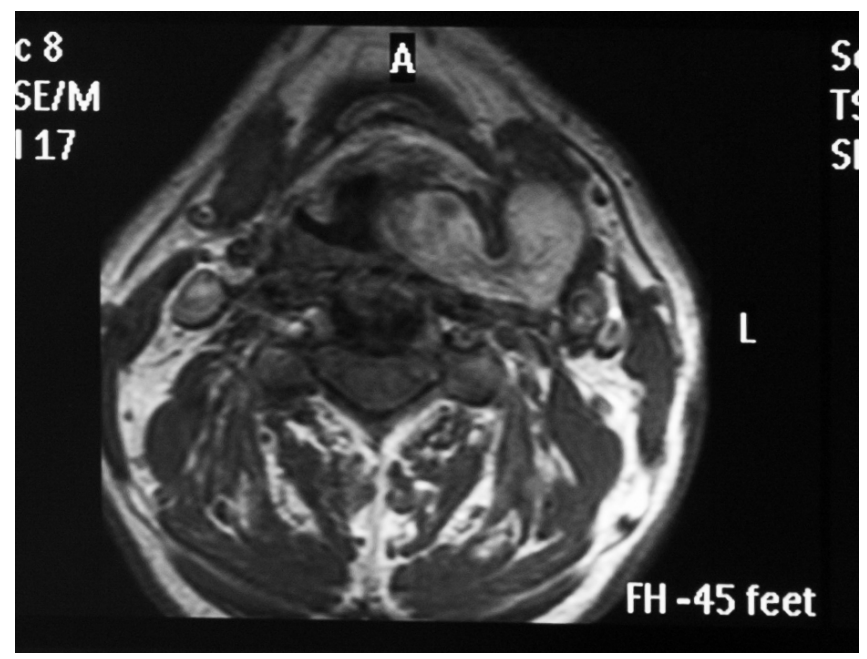

Figure 2. An axial neck MRI showing a homogenous and well-defined encapsulated mass of the hypopharynx with an extension to the visceral space on the left side. 


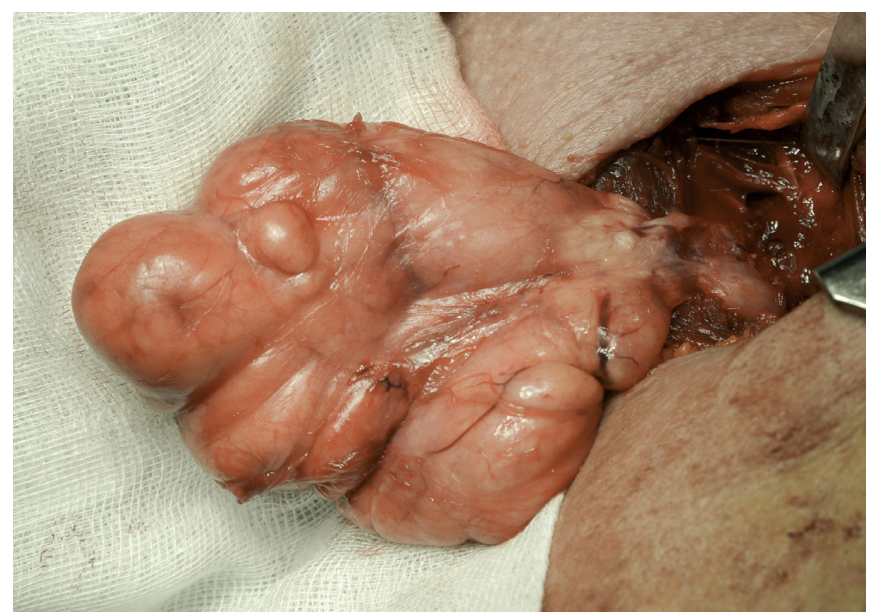

Figure 3. The tumour completely exposed and dissected through the left cervicotomy.

\section{Discussion}

Lipomas of the upper aerodigestive tract are composed of mature adypocites, histologically indistinctive from normal submucosal fat cells. The adipose tissue is relatively sparse in larynx and hypopharynx. It is mostly contained in the submucosa of the epiglottis, the aryepiglottic and ventricular folds. In this case the tissue involved was located in the hypopharyngeal and retropharyngeal submucosa. Macroscopically, hypopharyngeal lipomas appear as a smooth, submucosal swelling or, more frequently, as a pedunculated, polypoid lesion. ${ }^{2}$ Other pedicled or submucosal benign neoplastic entities of the hypopharynx, such as leiomyoma, other histologic subtypes of lipoma, pharyngeal polyps, papillomas or retention cysts should be considered in the differential diagnosis.

Patients with pharyngeal lipoma usually complain of mild and non-specific symptoms such as dysphagia, foreign body sensation and throat discomfort, or they may remain asymptomatic for a longer period of time until the lipoma reaches a larger size. In elongated polypoid lesions, the patient may present with lipoma protruding through the mouth, which is usually preceded by a coughing episode. ${ }^{2,3}$ The patient with pedicled lipoma may also experience episodes of breathing difficulties due to occasional lipoma prolapse in the laryngeal inlet. ${ }^{2}$ Death due to asphyxia has also been described. ${ }^{4}$ Our lesion, which was fairly large $(8 \mathrm{~cm})$ and with a wide base did not cause airway compromise or swallowing problems.

Diagnostic imaging, including CT or MRI, is an essential step in the evaluation of the hypopharyngeal mass. MRI with its superior soft-tissue contrast resolution provides a better tumour delineation and facilitate the treatment planning, especially in the case of larger lesions and infiltrating lipomas. ${ }^{5}$ In this case MRI was used to delineate the true extent of the lesion and to plan the surgical approach. The hypopharyngeal part of the tumour was approximately the same size as its extrapharyngeal part, so the precise site of origin could not be determined. The lesion was well-defined and without evidence of invasion of adjacent structures. 


\section{*Correspondence}

Ognjen Cukic

Department of Otorhinolaryngology

with Maxillofacial Surgery, Clinical

Hospital Centre Zemun

Vukova 9

11080, Zemun, Serbia

Tel.: +381 (11) 3772-660

E-mail: ognjen.cukic.bg@gmail.com

\section{Authors information}

OC - Doctor of Medicine (MD);

Otorhinolaryngologist at Department of Otorhinolaryngology with

Maxillofacial Surgery, Clinical Hospital

Centre Zemun. MJ - Doctor of

Medicine (MD); Otorhinolaryngologist;

$\mathrm{PhD}$ and Professor of

Otorhinolaryngology at Chair of

Otorhinolaryngology, University of

Belgrade.
The treatment of choice for hypopharyngeal lipoma is complete surgical excision. The transoral approach via the suspension laryngoscopy is usually sufficient for polypoid-like lipomas of hypopharynx with a narrow stalk and without involvement of the adjacent spaces. The transoral route is also adequate for more medially located retropharyngeal lipomas. In our case however, significant lateral extension of lipoma outside the hypopharynx required an open cervical approach to obtain complete removal and avoid recurrence. Lipoma usually consists of mature adipose tissue subdivided into lobules by a meshwork of fibrous strands and surrounded by a thin, fibrous capsule. In our case a distinct capsule was identified around the lipoma, which facilitated surgical removal. Transcervical approach provided us with an excellent exposure of the mass, allowing its complete removal, while carefully preserving the surrounding neural and vascular structures and maintaining integrity of the larynx. Postoperatively, the patient required a feeding tube for ten days, but no temporary tracheostomy was needed.

Hypopharyngeal lipoma is a rare lesion which necessitates a complete endoscopic and radiologic workup, as a significant extrapharyngeal spread may be present, even in clinically smaller submucosal lesions in the hypopharynx and without evident neck mass.

\section{References}

1. Enzinger FM, Weiss SW. Benign lipomatous tumours. In: Enzinger FM, Weiss SW, editors. Soft tissue tumours. 3rd ed. Saint Louis: Mosby; 1995. p. 381-430.

2. El-Monem MH, Gaafar AH, Magdy EA. Lipomas of the head and neck: presentation variability and diagnostic work-up. J Laryngol Otol. 2006;120(1):4755. PMid:16359147.

3. Acquaviva G, Varakliotis T, Badia S, Casorati F, Eibenstein A, Bellocchi G. Lipoma of piriform sinus: a case report and review of the literature. Case Rep Otolaryngol. 2016;2016:2521583. http://dx.doi.org/10.1155/2016/2521583. PMid:27795863.

4. Fyfe B, Mittleman RE. Hypopharyngeal lipoma as a cause of sudden asphyxial death. Am J Forensic Med Pathol. 1991;12(1):82-4. http://dx.doi.org/10.1097/00000433199103000-00016. PMid:2063825.

5. Cappabianca S, Colella G, Pezzullo MG, Russo A, laselli F, Brunese L, Rotondo A. Lipomatous lesions of the head and neck region: imaging findings in comparison with histological type. Radiol Med. 2008;113(5):758-70. http://dx.doi.org/10.1007/ s11547-008-0258-5. PMid:18414810. 